\title{
Lithography and Chemical Modeling of Acid Amplifiers for Use in EUV Photoresists
}

\author{
Seth Kruger, ${ }^{a}$ Craig Higgins, ${ }^{a}$ Gregg Gallatin, ${ }^{b}$ and Robert Brainard ${ }^{a}$ \\ a College of Nanoscale Science and Engineering, University at Albany, NY 12203 \\ $b$ Center for Nanoscale Science and Technology, National Institute of Standards \\ and Technology, Gaithersburg, MD 20899
}

\begin{abstract}
We postulate that the best way to simultaneously improve resolution, line edge roughness (LER), and sensitivity all in EUV resists is to increase the number of acid molecules generated per absorbed photon. In previous work, we showed that acid amplifiers (AAs) decompose auto-catalytically to effectively increase the amount of acid generated from each EUV photon.

In this paper, we show that AAs can simultaneously give better sensitivity and lower LER to improve Z-Parameter up to $3 \mathrm{X}$ compared to resists without AA. Acid amplifiers must be thermally stable to prevent the creation of acid in unexposed regions of the resist film. We use thermally-programmed spectroscopic ellipsometry to measure the decomposition of AAs in resist films and to identify structural features that influence the stability of AAs.

We also present the construction and utilization of a mathematical model that describes acid diffusion and acid amplifier decomposition in resist films. Using this model, we explored how the acid gradient between exposed and unexposed regions of a resist film is altered as a function of several variables; resist quantum yield, acid and base diffusion, AA and base loading, and AA decomposition kinetics. Out of the variables that were investigated, the model results are in general agreement with experimental results and demonstrate the potential for AAs to simultaneously improve resolution, LER and sensitivity.
\end{abstract}

Keywords: Acid amplifier, EUV, photoresists, diffusion model, Z-Parameter

\section{Introduction}

Extreme ultraviolet (EUV) lithography continues to be a strong candidate for a commercially viable solution to next generation lithography. However, further development of chemically amplified photoresists for use with EUV is critical to meet the future photolithographic requirements of the microelectronics industry. EUV resists must simultaneously meet three requirements: high resolution (below $22 \mathrm{~nm}$ ), low line edge roughness (LER), [1] and high sensitivity [2]. We have proposed that the best way to simultaneously improve these three properties in EUV resists is to increase the number of strong acids generated per photon absorbed during exposure [3] and we assert that acid amplifiers may be one of the best ways to achieve this goal. Acid amplifiers (AAs) are compounds that decompose in the presence of acid to generate more acid via acid-catalyzed mechanisms [4]. When the product acid is strong enough to catalyze the decomposition of the AA, the decomposition occurs autocatalytically (Figure 1) [5]. Based on this definition, the chemical structure of AA molecules should contain an acid sensitive functional group and an acid precursor. Our synthetic efforts focus on designing thermally stable AAs that produce fluorinated sulfonic acids for use in phenolic EUV resists.

In this work, we vary the chemical structures of our AAs to give a range of reactivities. These acid amplifiers consist of three parts (Figure 1), a body, an acid-sensitive trigger ( $\mathrm{T}$, either hydroxyl, methoxy, acetate or ketal), and a sulfonic acid precursor (A). Figure 2 shows two decomposition mechanisms that can occur to produce an acid. The undesirable decomposition pathway is uncatalyzed (U) thermal 
decomposition which results in the formation of an olefin byproduct and an acid. The desired acid generation pathway is via acid catalyzed decomposition. During autocatalysis, the trigger undergoes acidolysis yielding an allylic sulfonic ester. This olefin intermediate allows the sulfonic ester to thermally decompose via an $\mathrm{E}_{1}$ or $\mathrm{E}_{2}$ elimination reaction more rapidly than the starting $\mathrm{AA}$, yielding a second double bond alkene fragment and a sulfonic acid.

Several acid amplifiers have been reported in the literature. The reactivity of these compounds is highly dependent upon the structure of the body, trigger and acid precursor [6]. Triggers that have been studied are alcohols [7-9], ketals [10], carbonates [11] and tert-alkylcarboxy groups [12]. The acid generated by these compounds is almost always a sulfonic acid, and typically toluene sulfonic acid. Only two acid amplifiers previously reported in the literature produce fluorinated sulfonic acids [13]. The AAs presented in the literature do not meet the requirements for use in EUV photoresists.

In this paper we focus on the development and utilization of acid amplifiers for EUV resists. We measured AA thermal stability in resist films, evaluated the additive effects of AAs on resist lithographic performance and developed a model that predicts AAs can improve resist sensitivity and LER.

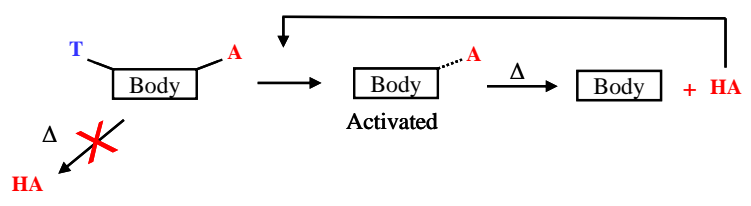

Figure 1. Generic representation of acid amplifiers (AAs). These compounds consist of three parts: a trigger $(\mathrm{T})$, an acid precursor (A) and a body.

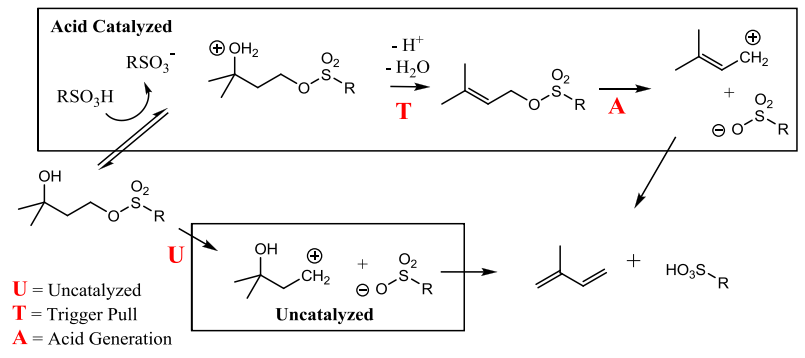

Figure 2. Proposed mechanism for acid catalyzed and uncatalyzed acid amplifier (AA) decomposition.

\section{AA Thermal Stability in Resist Films}

We used thermally-programmed spectroscopic ellipsometry [14] to quantitatively measure AA thermal decomposition temperatures. Thermally- programmed spectroscopic ellipsometry was used to measure the resist film thickness as a function of temperature. When AAs thermally decompose they release an acid which deprotects the resist polymer causing isobutene to outgas. The outgassed isobutene causes a decrease in the measurable film thickness. Acid amplifier decomposition temperatures $\left(T_{\mathrm{d}}\right)$ in resist films were quantified using this method.

Thermally programmed spectroscopic ellipsometry was used to measure the decomposition temperatures of our $\boldsymbol{O S} \boldsymbol{1}$ control resist (no AA) [15] and $\boldsymbol{O S} \boldsymbol{1}$ prepared with $70 \mathrm{mmol} / \mathrm{L}$ of added $\mathbf{3 H B}, \mathbf{3 H F}, \mathbf{3 M B}$ and $\mathbf{3 H G}$ acid amplifiers. Resist films were spin coated onto silicon substrates and soft baked at $90{ }^{\circ} \mathrm{C}$ for $60 \mathrm{~s}$. The resist film thickness was monitored as the samples were heated at a temperature ramp rate of $10^{\circ} \mathrm{C} / \mathrm{min}$.

Figure $3 \mathrm{~A}$ shows the normalized film thickness (initial film thickness is $70 \pm 3 \mathrm{~nm}$, final film thickness is $60 \pm 3$ $\mathrm{nm}$, Note: All uncertainties in this paper are $\approx \pm 3$ standard deviations.) as a function of temperature. The rapid decrease in film thickness is the result of isobutene outgassing. At these relatively low temperatures, the formation of isobutene requires an acid catalyst, which comes from AA decomposition. We define the decomposition temperature from the steepest part of the curve. We determine the decomposition temperature by plotting the derivative of film thickness versus temperature (Figure 3B). The decomposition temperatures of $\mathbf{O S 1}, \mathbf{3 H B}, \mathbf{3 H F}, \mathbf{3 M B}$ and $\mathbf{3 H G}$ are approximately $(195,152,139,128,112){ }^{\circ} \mathrm{C}$, respectively. Based on the calibration of the thermocouple used to measure the temperatures their uncertainty is on the order of $\pm 2{ }^{\circ} \mathrm{C}$. It should be noted that it is the relative values of the temperature which are important here and not their absolute values.

These AA thermal decomposition results show that the trigger and acid precursor contribute significantly to the thermal stability of AAs. Compounds $\mathbf{3 H B}$ and $\mathbf{3 M B}$ differ only by their trigger, yet $\mathbf{3 H B}\left(T_{\mathrm{d}}=152{ }^{\circ} \mathrm{C}\right)$ is $24{ }^{\circ} \mathrm{C}$ more stable than $\mathbf{3 M B}\left(T_{\mathrm{d}}=128{ }^{\circ} \mathrm{C}\right)$. This suggests that the methoxy trigger is more thermally labile than the hydroxyl trigger. Compounds $\mathbf{3 H B}, \mathbf{3 H F}$ and $\mathbf{3 H G}$ all have the same trigger but differ by their acid precursor type. The results suggest that the acidity of the liberated AA acid correlates with the AA thermal stability. The AA decomposition temperature decreases as the acid strength increases. This is a major challenge for 
designing new AAs because our goal is to make AAs that have high thermal stabilities and generate highly fluorinated super acids.

Thermal Decomposition of Acid Amplifiers In EUV Resist Films
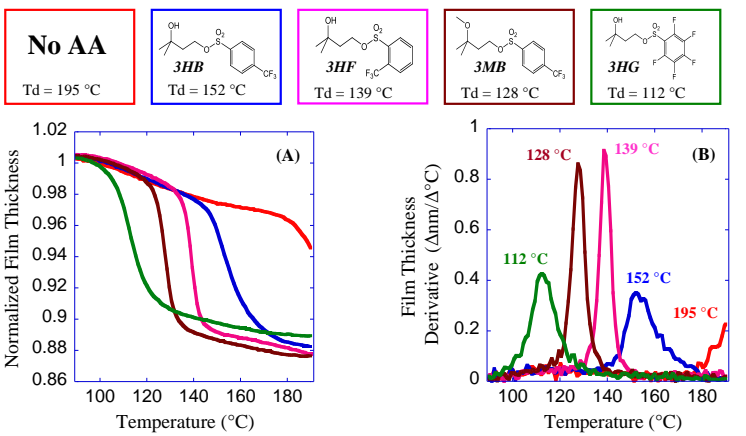

Figure 3. (A) Isobutene outgassing causes a sudden decrease in film thickness. (B) AA chemical structure affects the decomposition temperature. The uncertainties in the temperature values are about $\pm 2{ }^{\circ} \mathrm{C}$.

\section{Z-Parameter as a Function of Temperature}

Resist process conditions, particularly the post-exposure bake (PEB) temperature can have a large effect on resist sensitivity, LER and resolution. For example, increasing the PEB temperature improves the sensitivity by increasing the acid catalytic activity and diffusion length. However, this does not break the resolution-LER-sensitivity (RLS) trade-off because LER and resolution are degraded when acids diffuse farther. Therefore, the RLS trade off can not be broken by changing process conditions and so the Z-Parameter [16] should be independent of PEB temperature.

On the other hand, the effects of PEB temperature on resists with AAs are not as well understood. We investigated the effects of PEB temperature on resist performance because AA acid generation is dependent on PEB temperature. We expect AAs to generate more acid at higher PEB temperatures. We compared the $\boldsymbol{O S} \boldsymbol{1}$ control resist with $\boldsymbol{O S} \mathbf{1}$-type resists prepared with $70 \mathrm{mmol} / \mathrm{L}$ of $\mathbf{3 H B}$ or $\boldsymbol{6 A \boldsymbol { B }}$ at three $\mathrm{PEB}$ temperatures $(90,110,130){ }^{\circ} \mathrm{C}$. The Z-Parameter of OS1 does is independent of PEB temperature but the Z-Parameters of resists with added $\mathbf{3 H B}$ or $\mathbf{6 A B}$ improve significantly at lower temperatures (Figure 4). The largest improvement in Z-Parameter occurs at low PEB temperatures. Using a PEB of $90{ }^{\circ} \mathrm{C}$, the Z-Parameter of $\boldsymbol{O S} \boldsymbol{1}$ improves from $7.4 \times 10^{-7} \mathrm{~mJ} \cdot \mathrm{nm}^{3}$ to $5.4 \times 10^{-7} \mathrm{~mJ} \cdot \mathrm{nm}^{3}$ and $2.5 \times 10^{-7} \mathrm{~mJ} \cdot \mathrm{nm}^{3}$ for $\mathbf{3 H B}$ and $\mathbf{6 A B}$, respectively. The Z-Parameter uncertainties are $\approx \pm 25 \%$ which follows from the uncertainty in
LER $( \pm 0.5 \mathrm{~nm})$, Dose $( \pm 10 \%)$ and resolution $( \pm 0.5$ $\mathrm{nm})$.

Figure 5 shows the image quality of $\boldsymbol{O S I}$ with and without $\boldsymbol{\sigma A B}$. The soft bake and PEB conditions were $90{ }^{\circ} \mathrm{C}$ for $60 \mathrm{~s}$ and $90{ }^{\circ} \mathrm{C}$ for $90 \mathrm{~s}$ respectively. The dense line/space resolution limit of $\boldsymbol{O S \boldsymbol { 1 }}$ is $\approx 38 \mathrm{~nm}$ but with $\boldsymbol{G A B}, \approx 32 \mathrm{~nm}$ lines/spaces are resolved. $\boldsymbol{6} \boldsymbol{A} \boldsymbol{B}$ also improves the sizing dose $\left(E_{\text {size }}\right)$ of $\boldsymbol{O S} \boldsymbol{S}$ at $38 \mathrm{~nm}$ by $\approx$ $30 \%$, from $21.7 \mathrm{~mJ} / \mathrm{cm}^{2}$ to $16.6 \mathrm{~mJ} / \mathrm{cm}^{2}$ and improves LER by $\approx 75 \%$, from $8.6 \mathrm{~nm}$ to $4.9 \mathrm{~nm}$. These simultaneous improvements in resolution, LER and sensitivity are commensurate with the improvements in the Z-Parameters.

\section{Structure-Function Studies of Twelve AAs.}

To better understand the relationship between the structural features of acid amplifiers and their lithographic properties, we prepared and lithographically evaluated twelve AAs with variations in body, trigger, and acid precursors (Figure 6). Resists were prepared by adding $70 \mathrm{mmol} / \mathrm{L}$ AAs to the $\mathbf{O S 3}$ control resist [17] at a mass fraction of $5 \%$ solids (vs. total resist). Resists were spin coated to a film thickness of $125 \mathrm{~nm}$ and soft-baked $\left(90{ }^{\circ} \mathrm{C}, 60 \mathrm{~s}\right)$. Exposures were performed using dense line/space patterns (annular illumination on the Berkeley EUV micro-field exposure tool (MET)), followed by PEB $\left(90{ }^{\circ} \mathrm{C}, 90 \mathrm{~s}\right)$, followed by $45 \mathrm{~s}$ development in an aqueous solution of $0.26 \mathrm{~mol} / \mathrm{L}$ tetramethylammonium hydroxide (TMAH).

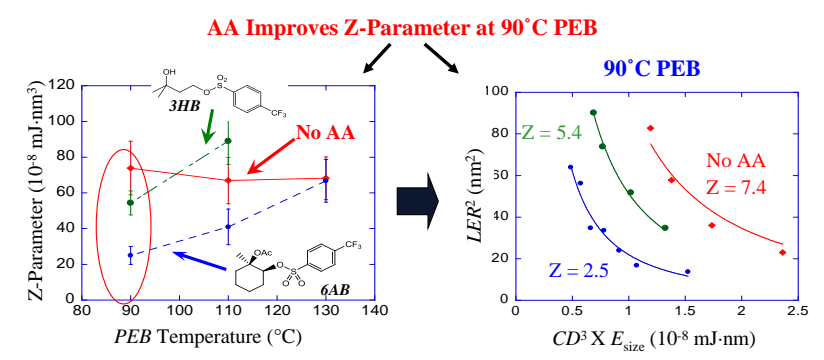

Figure 4. Z-Parameter for resist with out and with $70 \mathrm{mmol} / \mathrm{L}$ of $3 \mathrm{HB}$ or $6 \boldsymbol{A B}$. LER uncertainty is $\pm 0.5 \mathrm{~nm}$. Dose uncertainty is $\pm 10 \%$; Z Z Z-Parameter $/\left(10^{-7} \mathrm{~mJ} / \mathrm{cm}^{2}\right)$.

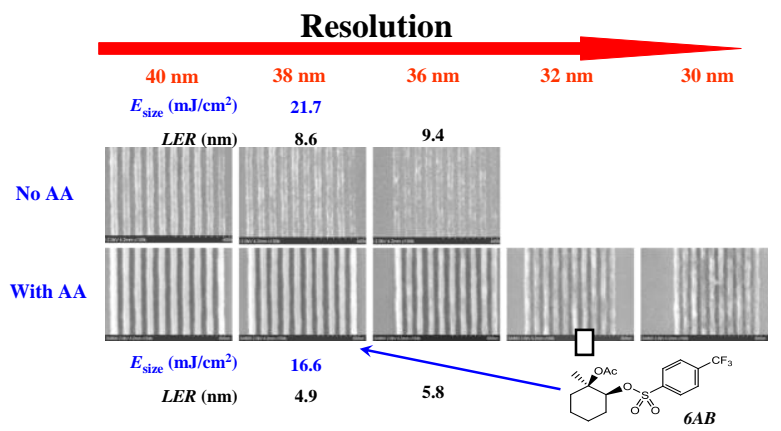

Figure 5. Scanning electron microscope (SEM) images of line/spaces pattern for $\boldsymbol{O S} \boldsymbol{1}$ control resist and $\boldsymbol{O S 1}$ with 70 $\mathrm{mmol} / \mathrm{L} \boldsymbol{6} \boldsymbol{A} \boldsymbol{B}$. LER uncertainty is $\pm 0.5 \mathrm{~nm}$. Dose uncertainty is $\pm 10 \%$. Resolution uncertainty is $\pm 0.5 \mathrm{~nm}$ 
The Z-Parameter was compared at $50 \mathrm{~nm}$ equal lines and spaces. A smaller Z-Parameter reflects overall improvement in lithographic performance. Figure 6 shows lithographic results for the control resist without an $\mathrm{AA}$ and twelve resists with 70 $\mathrm{mmol} / \mathrm{L}$ of AA. The sizing dose, LER and Z-Parameter are reported for each resist. All resists prepared with a $70 \mathrm{mmol} / \mathrm{L}$ concentration of AAs show sensitivity improvements (except $\mathbf{1 1 H F}$ ). The best overall resists contain AAs $\mathbf{3 M B}, \mathbf{3 M F}, \mathbf{3 H F}$ or $\mathbf{1 1 M B}$. The Z-Parameter of resists that contain $70 \mathrm{mmol} / \mathrm{L}$ of either of these AAs improves by factors of two or three relative to the control resist (control Z-Parameter $=13$ $\times 10^{-7} \mathrm{~mJ} \cdot \mathrm{nm}^{3}$ ). Addition of $\mathbf{3 H B}, \mathbf{1 1 H B}$ or $\mathbf{1 1 H G}$ improves the Z-Parameter from $13 \times 10^{-7} \mathrm{~mJ} \cdot \mathrm{nm}^{3}$ (control) to $7 \times 10^{-7} \mathrm{~mJ} \cdot \mathrm{nm}^{3}$ but AAs $\mathbf{1 1 M F}$ and $\mathbf{1 1 H F}$ do not improve the Z-Parameter. Figure 7 shows representative scanning electron micrographs of $50 \mathrm{~nm}$ lines and spaces for the control resist (no AA) and resists with $70 \mathrm{mmol} / \mathrm{L}$ of added tertiary AAs $(\mathbf{3 H B}$ or $3 M B)$ and secondary AAs $(11 \mathrm{HB}, 11 \mathrm{MB})$. Both secondary and tertiary AAs are capable of yielding lithographic improvements in sensitivity and LER.

Three of the four resists prepared with perfluorobenzenesulfonate ester AAs $(\mathbf{3 H G}, \mathbf{3 M G}$ or $11 M G)$ gave poorer lithographic performance vs. the control, so they were evaluated at $60 \mathrm{~nm}$ or $80 \mathrm{~nm}$ line/space (L/S). Thermal stability analysis showed that these AAs decompose between $70{ }^{\circ} \mathrm{C}$ and $110{ }^{\circ} \mathrm{C}$ so their poor lithographic performance may be due to their poor thermal stability. Interestingly, the only AA with a perfluorobenzenesulfonate ester that gave good lithographic performance is $\mathbf{1 1 H G}$ which has a secondary hydroxyl trigger. This AA resulted in the greatest improvement in resist sensitivity with only a modest degradation in LER.

Seven of the AAs were capable of showing improved lithographic performance (lower Z-Parameter) vs. the control resist. The Z-Parameter improved 3 -fold with the addition of $\mathbf{3 M B}$, the best improvement for the twelve AAs presented here. We speculate that $\mathbf{3 M B}$ gives the best lithographic improvements because of the combination of three attributes; it decomposes autocatalytically, generates the slowest diffusing acid and releases methanol as a byproduct.

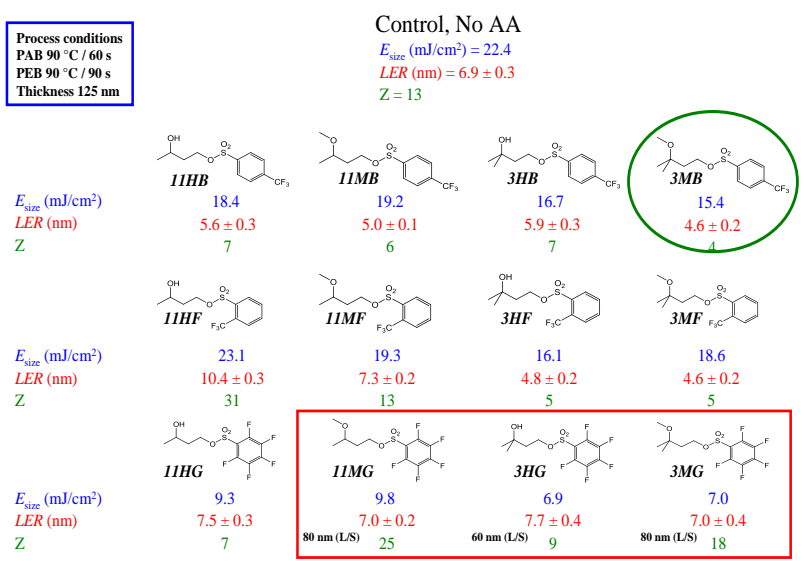

Figure 6. We exposed one control resist without AA and twelve resists with $70 \mathrm{mmol} / \mathrm{L} \mathrm{AA}$ to EUV light. The sizing dose $\left(E_{\text {size }}\right)$, LER and Z of these resists are reported for $50 \mathrm{~nm}$ equal lines and spaces (L/S) or at best resolution. $\mathrm{Z}=$ Z-Parameter $/\left(10^{-7} \mathrm{~mJ} / \mathrm{cm}^{2}\right)$. LER uncertainty is $\pm 0.5 \mathrm{~nm}$. Dose uncertainty is $\pm 10 \%$

\section{Acid Diffusion Model}

We developed a mathematical model to predict how acid amplifiers affect the lithographic performance of EUV photoresists. In the absence of AAs, increasing the amount of base quencher in a resist improves the LER. One explanation for this improvement is that the base increases the chemical contrast (acid gradient) at the line edge. We developed a model that simulates photo-acid generator (PAG) and AA acid and allows the acids and base quencher to diffuse as a function of time.

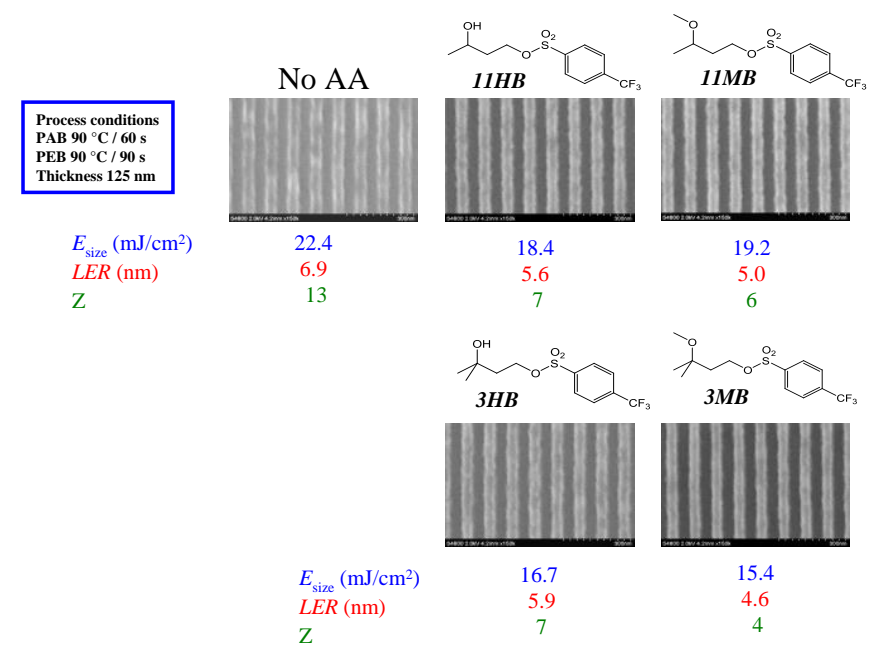

Figure 7. Scanning electron micrographs showing $50 \mathrm{~nm}$ dense lines of the control resist (no AA) and resists with $70 \mathrm{mmol} / \mathrm{L}$ of added $\mathbf{1 1 H B}$, $11 \mathrm{MB}, \mathbf{3 H B}$ or $\mathbf{3 M B}$. LER uncertainty is $\pm 0.5 \mathrm{~nm}$. Dose uncertainty is $\pm 10 \%$. Resolution uncertainty is $\pm 0.5 \mathrm{~nm}$. $\mathrm{Z}=\mathrm{Z}$-Parameter $/\left(10^{-7}\right.$ $\mathrm{mJ} / \mathrm{cm}^{2}$ ) 
Figure 8 shows an overview of how the model is divided into four calculable components: PAG photolysis, base quenching, acid and base diffusion and AA decomposition. Using this simple model, we compared the acid gradient at a line edge for various resist formulations. Additionally, we explored the acid gradient as a function of quantum yield $(Q Y), \mathrm{AA}$ and base loadings, acid and base diffusion lengths and AA reactivity. Our aim is to predict the relative combinations of AAs and base that produce the best sensitivity and steepest acid concentration gradients.

\section{Model Overview}

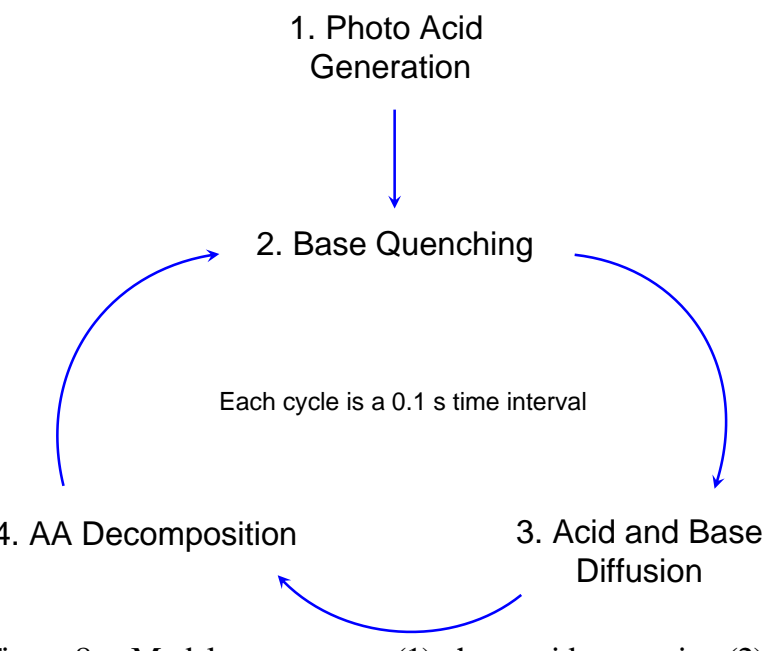

Figure 8. Model components, (1) photo acid generation (2) base quenching (3) diffusion (4) AA decomposition.

\subsection{Model Assumptions}

We made several simplifying assumptions to build this model. We think these assumptions will not affect any of the trends predicted by the model and assert that the benefits of these simplifications outweigh the accuracy of a more sophisticated model [18-21]. Simplifying the model allows us to apply the calculations to any resist formulation and process condition. The assumptions are: (1) acid from the PAG and AA are the same, (2) AAs are homogenously dispersed and do not diffuse, (3) acid does not outgas, (4) base quenching reaction is instantaneous, (5) diffusion is the same in exposed and unexposed regions, (6) concentration gradients are only in 1 dimension (7) quantum yield does not change as PAG is consumed.

\subsection{Correlating Model Results to Resist Formulations}

One caveat about acid, base and AA concentrations must be addressed in order to compare simulation results with experimental resist formulations. This model is not intended to make direct comparisons with experimental resist formulations but is instead designed to predict trends. We, therefore, used some approximations that allowed us to relate the model results to actual resist formulations.

We chose to have quantum yield $(Q Y)$ as an input variable because it contributes significantly to a resist's performance [22]. However, many variables affect the $Q Y$, such as resist composition and PAG loading, for this reason the PAG loading is not an input variable. As a reference point, the quantum yields for our resist with di(4-tert-butylphenyl) iodonium perfluoro-1-butanesulfonate PAG loadings at mass fractions of $7.5 \%$ (123 $\mathrm{mmol} / \mathrm{L}), 15 \%(247 \mathrm{mmol} / \mathrm{L})$ and $30 \%(494 \mathrm{mmol} / \mathrm{L})$ are 2.6, 3.8 and 5, respectively [22]. The base loadings that we use in our resist formulations range from mass fractions of $0.5 \%$ to $2 \%(22 \mathrm{mmol} / \mathrm{L}$ to $88 \mathrm{mmol} / \mathrm{L})$ and the AA loadings range from $0 \mathrm{mmol} / \mathrm{L}$ to $280 \mathrm{mmol} / \mathrm{L}$. The model uses the relative concentrations of PAG, base and AA instead of the exact amounts. Therefore, in the following sections all the concentration values derived from the model are relative values and hence are unitless unless explicitly stated otherwise. Given the relative values and the actual $(\mathrm{mol} / \mathrm{L})$ of any one component the real values of all the components can easily be determined.

\subsection{Model Equations}

The first step in building this model is to determine the initial acid concentration profile from PAG photolysis through a dense line/space mask. We assume that the aerial image intensity will have a sinusoidal shape with the peak intensity at the center of the transparent regions of the mask and zero intensity at the center of the opaque regions. Figure 9 illustrates the aerial image profile relative to the mask pattern. The amplitude of the aerial image is a function of exposure dose $\left(\mathrm{mJ} / \mathrm{cm}^{2}\right)$ and the acid concentration is calculated by multiplying the aerial image intensity with film quantum yield (moles of acid generated / moles of photons absorbed). The initial acid concentration is given by

$$
C_{x, t=0}=\left(\frac{Q Y \times \text { Dose }}{10}\right)\left(\cos \left(\frac{\pi x}{l}\right)+1\right)
$$

Here $C$ is the acid concentration, $x$ is position (nm), $l$ is feature size (nm), $t$ is time (s), $Q Y$ is quantum yield and Dose is the exposure dose $\left(\mathrm{mJ} / \mathrm{cm}^{2}\right)$. After PAG photolysis, the acid concentration is reduced by the amount of base quencher [23]. 


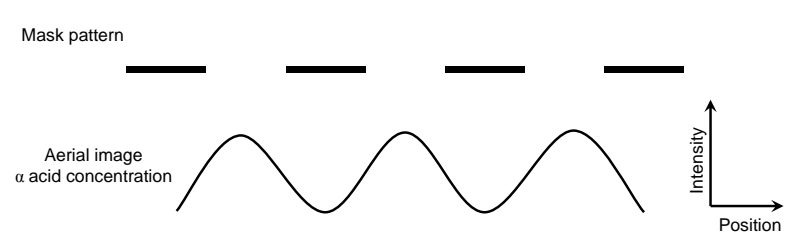

Figure 9. Aerial image for equal L/S mask pattern has a sinusoidal shape due to light diffraction.

The next step in the model is to calculate the concentration of acid and base as they diffuse. The main driving force for diffusion is the concentration gradient. The acid and base diffuse from high concentrations toward low concentrations at a rate proportional to the acid diffusion length $\sqrt{ }(2 D t)$. The acid and base concentrations at times $t>0$ were calculated by applying the standard finite difference scheme

$$
\begin{aligned}
C_{x, t}= & C_{x, t-\Delta t} \\
& -D \Delta t\left(\frac{2 C_{x, t-\Delta t}-C_{x+\Delta x, t-\Delta t}-C_{x-\Delta x, t-\Delta t}}{\Delta x^{2}}\right)
\end{aligned}
$$

to both concentrations. Here $D$ is the diffusivity, $\Delta x$ and $\Delta t$ are the position and time steps, respectively. The total number of time steps is then $t / \Delta t$.

If acid amplifier is present then the acid concentration is increased by the amount of acid generated from the AA decomposition, $P$ which was calculated using [24].

$$
P_{x, t}=\frac{A A_{x, t-\Delta t}+C_{x, t-\Delta t}}{1+\frac{A A_{x, t-\Delta t}}{C_{x, t-\Delta t}} \exp \left[-\left(A A_{x, t-\Delta t}+C_{x, t-\Delta t}\right) k \Delta t\right]}
$$

where $k\left(\mathrm{~s}^{-1}\right)$ is the AA decomposition rate constant.

Acid amplifier decomposes autocatalytically with rate constant $k$, and can only decompose when there is initial catalytic acid $\left(C_{x, t-\Delta t}>0\right)$. Acid from AA decomposition is added to the total acid concentration $C_{x, t} \rightarrow C_{x, t}+P_{x, t}$. It then diffuses the same as the PAG acid.

The last step in a single iteration is to subtract the base from the acid concentration with the constraint that the minimum allowable acid concentration is 0 ; this completes one time interval. The process of diffusion, AA decomposition and base quenching is repeated 1000 times for a total simulation time of 100 s.

Figure 10 helps illustrate acid diffusion by showing the acid concentration as a function of position at $0 \mathrm{~s}$,
$30 \mathrm{~s}, 60 \mathrm{~s}$ and $90 \mathrm{~s}$. For simplicity, this simulation does not contain base or AA. The top plot shows the acid concentration over the full range of the $60 \mathrm{~nm} \mathrm{~L} / \mathrm{S}$ mask pattern and the bottom plot focuses in from the center of the exposed region $(x=0 \mathrm{~nm})$ to the center of the unexposed region $(x=66 \mathrm{~nm})$ with the line edge between $x=30 \mathrm{~nm}$ and $x=36 \mathrm{~nm}$. At time 0 , the initial acid concentration profile (green curve) has a maximum of 3.9 (at $x=0 \mathrm{~nm}$ ) and a minimum of 0 (at $x=66 \mathrm{~nm}$ ). As time progress the acid diffuses from the high concentration regions to the low concentration regions and after $90 \mathrm{~s}$ the acid concentrations at $x=0 \mathrm{~nm}$ and $x=$ $66 \mathrm{~nm}$ are 3.5 and 0.3 respectively.
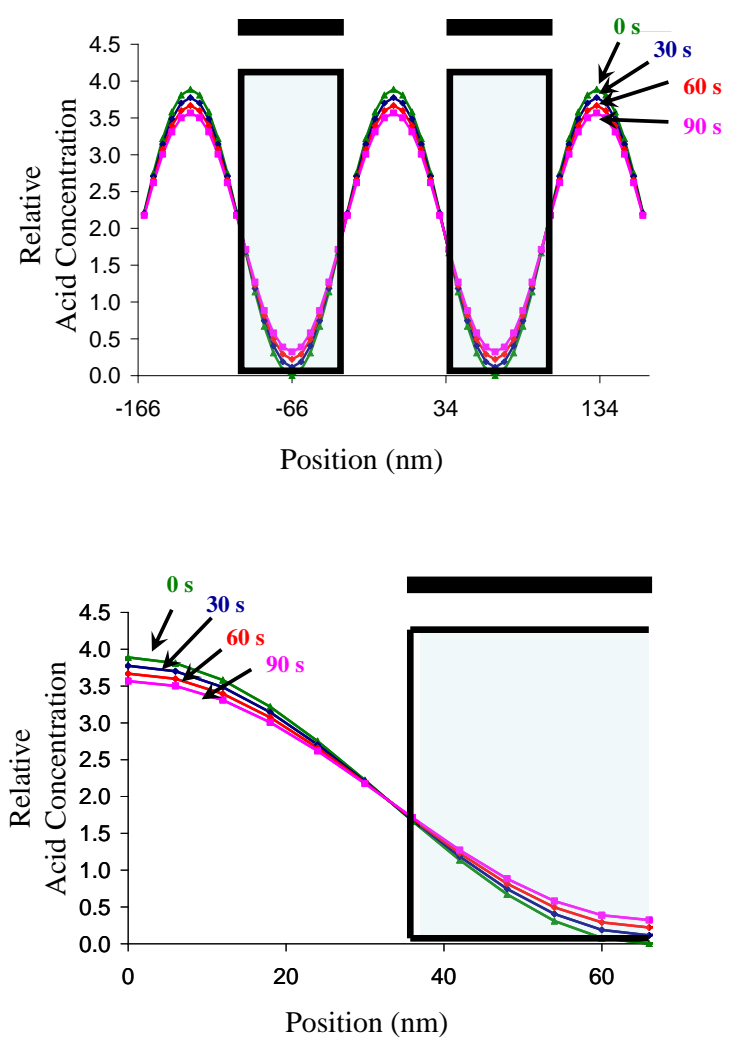

Figure 10. Simulation example without added base or AA showing the relative acid concentration (unitless) profile at 0,30 , 60 , and $90 \mathrm{~s}$.

Figure 11 illustrates key parameters that remain constant between simulated results. Experimental lithographic results are typically compared at a sizing dose under constant process conditions. The sizing dose is determined by the level of polymer deprotection, which is a function of acid concentration and reaction time [25]. Likewise, our simulation results are compared at a sizing dose and at a constant amount of diffusion time. We define the sizing dose as the dose required to reach an 
accumulated acid concentration of 200 at the desired line edge position at $90 \mathrm{~s}$. Resist LER is influenced by the chemical contrast at the line edge [26], so the analogous figure of merit that we compare between simulations is the accumulated acid concentration gradient at the line edge (red dashed line); a steeper gradient indicates a better performing resist.

5.4. Acid Gradient vs. Quantum Yield and Base Loading

This set of calculations was designed to determine the effects of quantum yield $(Q Y)$ on sensitivity and acid gradient at a constant base loading of 0.5. The acid and base diffusivities $(D)$ are both $1 \mathrm{~nm}^{2} / \mathrm{s}$. We chose to vary the $Q Y$ from 1.99 to 5.09 because we can compare our model results with experimental data at these $Q Y \mathrm{~s}$.

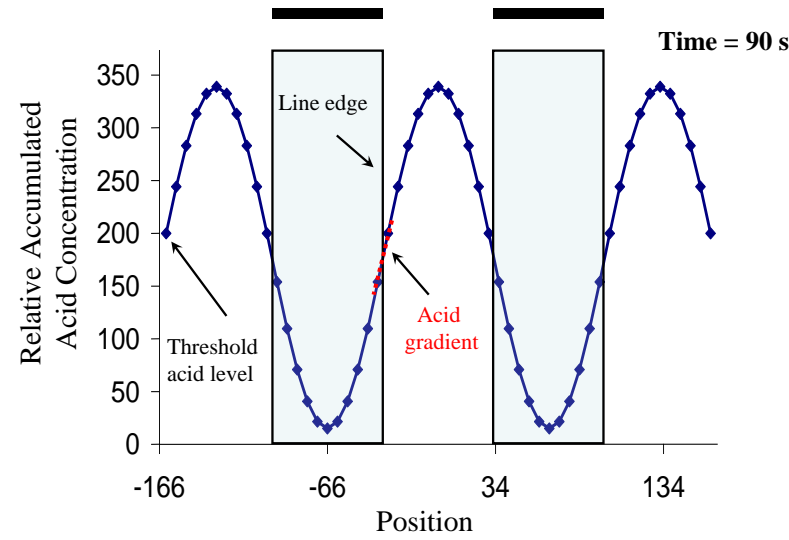

Figure 11. For consistency, model results are compared at a threshold relative accumulated acid concentration (unitless) of 200 at the line edge at $90 \mathrm{~s}$.

Figure 12 compares the experimental and calculated doses, LERs and acid gradients at various $Q Y$ s. Figure 13 shows the acid gradient and LER plotted as a function of $Q Y$. Experimentally, we have previously shown that increasing $Q Y$ directly improves the sensitivity while the LER essentially remains unchanged [22]. The model also shows the same trend, increasing $Q Y$ improves sensitivity but has no effect on the chemical gradient.

The next set of calculations compares the effect of base loading on the sensitivity and acid gradient for various $Q Y$ s. Figure 14 shows the accumulated acid concentration gradient plotted as a function of dose. Each set (same color) of data points represents a different $Q Y$ ranging from 2 to 5. Each point within a set corresponds to a different base loading from 0 to 3 in increments of 0.5 . As expected, the dose increases as base loading increases,

\begin{tabular}{|c|c|c|c|c|}
\cline { 2 - 5 } \multicolumn{1}{c|}{} & \multicolumn{2}{c|}{ Experimental } & \multicolumn{2}{c|}{ Model } \\
\hline$Q Y$ & $\begin{array}{c}\text { Dose } \\
\left(\mathrm{mJ} / \mathrm{cm}^{2}\right)\end{array}$ & $\begin{array}{c}\text { LER } \\
(\mathrm{nm})\end{array}$ & $\begin{array}{c}\text { Dose } \\
\left(\mathrm{mJ} / \mathrm{cm}^{2}\right)\end{array}$ & $\begin{array}{c}\text { Gradient } \\
(1 / \mathrm{nm})\end{array}$ \\
\hline 1.99 & 16.9 & 4.5 & 12.0 & 9.1 \\
\hline 2.61 & 10.3 & 4.6 & 9.1 & 9.1 \\
\hline 3.84 & 5.9 & 4.6 & 6.2 & 9.1 \\
\hline 4.39 & 5.1 & 4.2 & 5.4 & 9.1 \\
\hline 5.09 & 4.3 & 4.5 & 4.7 & 9.1 \\
\hline
\end{tabular}

Figure 12. Experimental and calculated sensitivities improve as quantum yield increases.

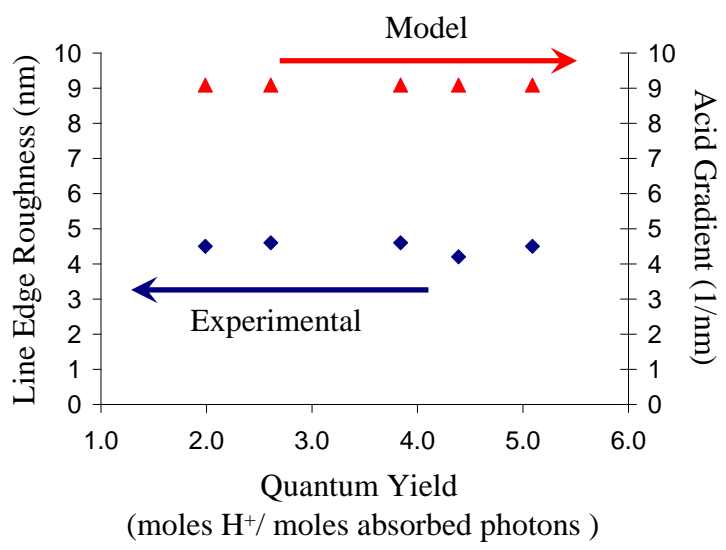

Figure 13. ( $\bullet$ Experimental LER and ( $\mathbf{\Delta})$ calculated acid gradient are constant as a function of quantum yield.

however the dose decreases as the $Q Y$ increases (at a constant base loading). An interesting comparison to make is with the acid gradient at a constant dose. For example, at $10 \mathrm{~mJ} / \mathrm{cm}^{2}$, the acid gradient improves as $Q Y$ increases; however the base loading is also increased to maintain a constant dose. This result predicts that a resist's LER performance can be improved by increasing the $Q Y$ and base loading without penalty to sensitivity and this has been confirmed experimentally [22].

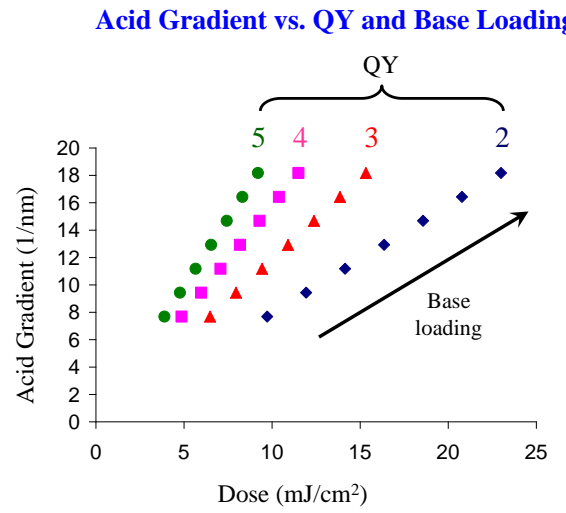

Figure 14. Acid gradient as a function of quantum yield and base loading. 
5.5. Acid Gradient vs. AA and Base Loading

This set of calculations was designed to determine the effects of acid amplifier and base loadings on sensitivity and acid gradient at a constant $Q Y$ of 2.5 . The acid and base diffusivities $(D)$ are both $1 \mathrm{~nm}^{2} / \mathrm{s}$ and the AA decomposition rate constant $(k)$ is $0.02 \mathrm{~s}^{-1}$. Figure 15 shows the acid gradient plotted as a function of dose. Each set of data (same color) corresponds to a different base loading, 0, 0.5, 1.0, 1.5, 2.0, and 3.0. Each data point within a set is a different AA loading. At 0 base loading, the AA loadings are 0, 0.5, 1.0, 1.5, 2.0, 2.5, 3.0 and 4.0; for all other base loadings the AA loadings are $0,0.5,1.0,1.5,2.0,2.5,3.04 .0,5.0,6.0$ and 7.0. As expected, the dose increases with increasing base loading. At all base loadings the addition of AA improves the sensitivity but does not always improve the acid gradient. In the absence of base, AA only makes the acid gradient worse. At base loadings of 0 and 1.0, low AA degrades the acid gradient but improves the gradient at higher AA loadings. The addition of AA when the base loading is 1.5 and higher improves the acid gradient. This result predicts that AAs can simultaneously improve the sensitivity and acid gradient at the proper base and AA loadings.

Acid Gradient vs. Base and AA Loading

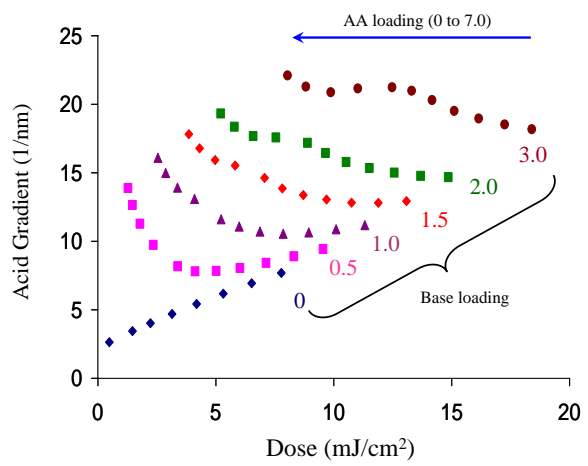

Figure 15. Acid gradient as a function of acid amplifier and base loading.

The model predicts that $Q Y$ and AA both have the potential to improve the sensitivity and acid gradient. We calculated an analogous Z-Parameter value using the model results to compare the overall benefits of increasing $Q Y$ with the benefits of using AAs. Figure 16 shows the equation used to calculate the Z-Parameter. The model Z-Parameter equation uses $1 /$ (acid gradient) $^{2}$ as opposed to $L E R^{2}$ because the acid gradient is inversely correlated to LER. The model Z-Parameter is calculated for three resists, one with AA
(C) and two without (A and B). Figure 17 tabulates the model Z-Parameter results for the three resists. Resist A has a $Q Y$ of 2.5 and Z-Parameter of 23.2. Increasing the $Q Y$ to 5.0 (resist B) and adding base to maintain a nearly constant dose improves the Z-Parameter by 3.9X. We chose a $Q Y$ of 5.0 because that is the maximum $Q Y$ experimentally achieved (for a usable resist) with our resist formulation. Resist $\mathrm{C}$ has the same $Q Y$ as resist $\mathrm{A}$, but contains acid amplifier and a higher base loading to keep the dose nearly constant. Resist $\mathrm{C}$ improves the Z-Parameter by $4.7 \mathrm{X}$ (versus resist $\mathrm{A}$ ). This result is consistent with experimental evidence that that the overall resist performance can be improved by either increasing QY or through the addition of acid amplifiers.

\section{$(\text { Half Pitch })^{3} \times(\text { Acid Gradient })^{-2} \times$ Esize $=$ Z-Parameter}

Figure 16. Model Z-Parameter is used to calculate the overall resist performance.

\begin{tabular}{|c|c|c|c|c|c|c|}
\hline Resist & $Q Y$ & Base & AA & $\begin{array}{c}\text { Dose } \\
(\mathrm{mJ} / \mathrm{cm} 2)\end{array}$ & $\begin{array}{c}\text { Gradient } \\
(1 / \mathrm{nm})\end{array}$ & $\begin{array}{c}\text { Model } \\
\text { Z-Parameter } \\
\left(\mathrm{mJ}^{\prime} \cdot \mathrm{nm}^{3} \times 10^{3}\right)\end{array}$ \\
\hline $\mathrm{A}$ & 2.5 & 0.5 & 0.0 & 9.5 & 9.4 & 23.2 \\
\hline $\mathrm{B}$ & 5.0 & 3.0 & 0.0 & 9.2 & 18.2 & 6.0 \\
\hline $\mathrm{C}$ & 2.5 & 3.0 & 5.0 & 9.9 & 20.9 & 4.9 \\
\hline
\end{tabular}

Figure 17. Acid amplifiers improve the Z-Parameter more than increasing $Q Y$.

\subsection{Acid Gradient vs. AA Loading and Decomposition Rate Constant}

We synthesized many AAs that have a range of decomposition rates. Here, we calculate the acid gradient as a function of AA decomposition rate constant to help us understand the effects of AA decomposition kinetics on the acid gradient and sensitivity. Figure 18 shows acid gradient as a function of AA rate constant for two base loadings ( 0.5 and 3.0) and eight AA loadings (0 to 10). The calculations were done using autocatalytic rate constants of $0.007 \mathrm{~s}^{-1}(\boldsymbol{\Delta}), 0.02 \mathrm{~s}^{-1}(\diamond)$, and $0.04 \mathrm{~s}^{-1}(\mathbf{\square})$. The results show that AAs improve the sensitivity regardless of AA rate constant or base loading. However, AAs with high rate constants improve the sensitivity more than AAs with small rate constants. An unexpected finding from these calculations is that AAs with smaller rate constants give better acid gradients when all other variables are constant. This is most apparent at the higher base loading. Data for the AA decomposition rate constant of $0.04 \mathrm{~s}^{-1}(\mathbf{\square})$ shows the acid gradient is only improved from $18.2 \mathrm{~nm}^{-1}$ to $20 \mathrm{~nm}^{-1} \approx$ $10 \%$ ) as the AA loading is increased from 0 to 10 . On 
the other hand, the AA rate constant of $0.007(\mathbf{\Delta})$ shows an acid gradient improvement from 18.2 to 26.4 (45\%) over the same AA loading. These calculations predict that AAs with lower catalytic rate constants will provide the steeper acid gradients but with some loss in sensitivity. The largest gain in lithographic performance is predicted by using high concentrations of AAs that have low catalytic rate constants.

Acid Concentration Gradient vs AA Loading and AA Reactivity at Two Base Loadings

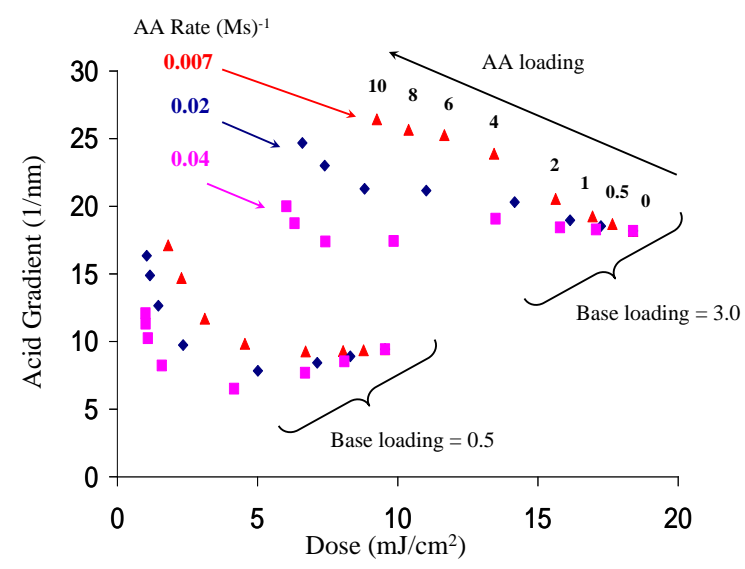

Figure 18. Acid gradient as a function of AA and base loading for three AAs with different decomposition rate constants

\section{Summary and Conclusions}

Acid amplifiers designed for use in EUV photoresists must have three critical properties. First, AAs must be thermally stable under resist process conditions. Second, AAs must rapidly decompose in the presence of catalytic acid. Third, AAs must generate strong (fluorine-containing sulfonic) acids capable of deprotecting the photoresist polymer.

In this work, we have presented acid amplifiers that generate moderately strong, fluorinated sulfonic acids. We synthesized AAs with systematic variations in chemical structure to give a range of reactivities. We used thermally-programmed spectroscopic ellipsometry to measure decomposition temperatures of AAs in resist films. Most importantly, we evaluated the lithographic performance of EUV resists with added AAs and demonstrated that some AAs can simultaneously improve resist resolution, LER and sensitivity.

We developed an acid diffusion model to predict AAs effects on resist lithographic performance. In general, the calculations show that optimizing the performance of a resist is complicated by many variables, such as diffusion lengths, formulation composition and reaction rates. Nonetheless, this model gives insight about how each variable affects the resist performance but requires more refinement to calibrate the calculated results with experimental results. The most important conclusion that we draw from the model results is that AAs can simultaneously improve the acid gradient and sensitivity at high base loadings, which is critical to beat the RLS-tradeoff. We hypothesize that these improvements are the result of two factors. First, AAs improve the sensitivity because they generate additional acid in the resist without increasing the dose. Second, AAs improve the acid gradient at the line edge because they release acid over the entire PEB time so newly generated acids have smaller diffusion domains and less chance of being neutralized by base.

\section{Acknowledgements.}

The authors of this paper would like to thank Intel Corporation for partial financial support of this research. In particular, we thank Todd Younkin, Wang Yueh, and E. Steve Putna for guidance and useful discussions. We also thank Chuck Szmanda for developing early versions of this simple excel modeling method and we thank Brian Cardineau for being an indispensible part of our team. Lastly, we thank DuPont for supplying us with photoresist polymers.

\section{References}

[1] M. D. Shumway, P. Naulleau, K. A. Goldberg, and J. Bokor, Journal of Vacuum Science \& Technology, B: Microelectronics and Nanometer Structures-Processing, Measurement, and Phenomena, 2005, 23(6), p. 2844-2847.

[2] G. Gallatin, M. Gregg P. Naulleau, D. Niakoula, R. L. Brainard, E. Hassanein, R. Matyi, J. Thackeray, K. Spear, and K. Dean, Proceedings of SPIE, Emerging Lithographic Technologies, 2008, 6921, p. 69211E/1-69211E/11.

[3] R. L. Brainard, P. Trefonas, J. H. Lammers, C. A. Cutler, J. F. Mackevich, A. Trefonas, and S. A. Robertson, Proceedings of SPIE, Emerging Lithographic Technologies, 2004, 5374, p. 74-85.

[4] K. Ichimura, Chemical Record, 2002, 2(1), p. 46-55.

[5] C. J. Gerdts, D. E. Sharoyan, and R. F. Ismagilov, Journal of the American Chemical Society, 2004, 126(20), p. 6327-6331. 
[6] S-W. Park, K. Arimitsu, and K. Ichimura, Macromolecular Rapid Communications, 2000, 21, p. 1050.

[7] S. Noguchi, K. Arimitsu, K. Ichimura, K. Kudo, T. Ohfuji, and M. Sasago, Journal of Photopolymer Science and Technology, 1997, 10, p. 315.

[8] O. T. Naito, M. Endo, H. Morimoto, K. Arimitsu, and K. Ichimura, Journal of Photopolymer Science and Technology, 1999, 12, p. 509.

[9] S-W. Park, K. Arimitsu, K. Ichimura, and T. Ohfuji, Journal of Photopolymer Science and Technology, 1999, 12, p. 293.

[10] K. Kudo, K. Arimitsu, H. Ohmori, H. Ito, and K. Ichimura, Chemistry of Materials, 1999, 11 p. 2119.

[11] H. Ito, and K. Ichimura, Macromolecular Chemistry and Physics, 2000, 201, p. 132.

[12] K. Arimitsu, K. Kudo, and K. Ichimura, Journal of the American Chemical Society, 1998, 120, p. 37.

[13] S. Lee, K. Arimitsu, S-W. Park, and K. Ichimura, Journal of Photopolymer Science and Technology, 2000, 13, p. 215.

[14] H. G. Tompkins, "A user's guide to ellipsometry," Academic Press, 1993, San Diego, CA

[15] OS1 is composed of a mass fraction of $7.5 \%$ of the photoacid generator bis(4-tert-butylphenyl)iodonium nonaflate the phenolic terpolymer [poly(hydroxystyrene)-(styrene)-(t-buylacrylate) 650/ $200 / 150 \mathrm{mmol} / \mathrm{mol}]$ and a mass fraction of $0.5 \%$ of tetrabutyl ammonium hydroxide.

[16] T. Wallow, C. Higgins, R. L. Brainard, K. Petrillo, W. Montgomery, C-S. Koay, G. Denbeaux, O. Wood, and Y. Wei, Proceedings of SPIE, Emerging Lithographic Technologies, 2008, 6921, p. 69211F/ $1-69211 \mathrm{~F} / 11$.

[17] OS3 is composed of, a mass fraction of $7.5 \%$ of the photoacid generator bis(4-tert-butylphenyl)iodonium nonaflate the phenolic terpolymer [poly(hydroxystyrene)-(styrene)-(t-buylacrylate) 650/ $200 / 150 \mathrm{mmol} / \mathrm{mol}$ ] and a mass fraction of $1.0 \%$ of tetrabutyl ammonium hydroxide.

[18] J. Nakamura, H. Ban, and A. Tanaka, Japanese Journal of Applied Physics, Part 1, 1992, 31(12B), p. 4294-300.

[19] E. Croffie, M. Cheng, and A. Neureuther, Journal of Vacuum Science \& Technology, B: Microelectronics and Nanometer Struc-tures, 1999, 17(6), p. 3339-3345.

[20] A. Philippou, T. Muelders, and E. Schoell, Journal of Micro/Nanolithography, MEMS, and MOEMS, 2007, 6(4), p. 043005/1-043005/11.

[21] J. J. Biafore, M. D. Smith, E. Van Setten, T. Wallow, P. Naulleau, D. Blankenship, S. A. Robertson, Y. Deng, and B. M. La Fontaine, Proceedings of SPIE, Extreme Ultraviolet Lithography, 2010, 7636, p. $76360 \mathrm{R} / 1-76360 \mathrm{R} / 10$.

[22] C. D. Higgins, C. R. Szmanda, A. Antohe, G. Denbeaux, J. Georger, and R. L. Brainard, Japanese Journal of Applied Physics, 2011, 50(3).

[23] W. D. Hinsberg, F. A. Houle, M. I. Sanchez, M. E. Morrison, G. M. Wallraff, C. E. Larson, J. A. Hoffnagle, P. J. Brock, and G. Breyta, Proceedings of SPIE, Advances in Resist Technology and Processing, 2000,3999, p. 148-160.

[24] Second-order catalyzed reaction kinetics were evaluated by fitting data to the autocatalytic rate equation. Capellos, Christos, and B. Bielski, "Kinetics Systems, Mathematical Description of Chemical Kinetics in Solution", Wiley-Interscience, New York (1972).

[25] T. H. Fedynyshyn, J. W. Thackeray, J. H. Georger, and M. D. Denison, Journal of Vacuum Science \& Technology, B: Microelectronics and Nanometer Structures, 1994, 12(6), p. 3888-3894.

[26] T. Kozawa, H. Oizumi, T. Itani, and S. Tagawa, Japanese Journal of Applied Physics, 2009, 48, p. 126004/1-126004/5. 